\title{
An Orbital Window into the Ancient Sun's Mass
}

\author{
Christopher Spalding ${ }^{1}$, Woodward W. Fischer ${ }^{2}$, and Gregory Laughlin ${ }^{1}$ (DD \\ ${ }_{1}^{1}$ Department of Astronomy, Yale University, New Haven, CT 06511, USA \\ ${ }^{2}$ Division of Geological and Planetary Sciences, California Institute of Technology, Pasadena, CA 91125, USA \\ Received 2018 October 1; revised 2018 November 16; accepted 2018 November 17; published 2018 December 11
}

\begin{abstract}
Models of the Sun's long-term evolution suggest that its luminosity was substantially reduced 2-4 billion years ago, which is inconsistent with substantial evidence for warm and wet conditions in the geological records of both ancient Earth and Mars. Typical solutions to this so-called "faint young Sun paradox" consider changes in the atmospheric composition of Earth and Mars, and, while attractive, geological verification of these ideas is generally lacking - particularly for Mars. One possible underexplored solution to the faint young Sun paradox is that the Sun has simply lost a few percent of its mass during its lifetime. If correct, this would slow, or potentially even offset, the increase in luminosity expected from a constant-mass model. However, this hypothesis is challenging to test. Here, we propose a novel observational proxy of the Sun's ancient mass that may be readily measured from accumulation patterns in sedimentary rocks on Earth and Mars. We show that the orbital parameters of the Solar System planets undergo quasi-cyclic oscillations at a frequency, given by secular mode $g_{2}-g_{5}$, that scales approximately linearly with the Sun's mass. Thus by examining the cadence of sediment accumulation in ancient basins, it is possible distinguish between the cases of a constant-mass Sun and a more massive ancient Sun to a precision of greater than about 1 percent. This approach provides an avenue toward verification, or of falsification, of the massive early Sun hypothesis.
\end{abstract}

Key words: Earth - planets and satellites: terrestrial planets - solar wind

\section{Introduction}

The Earth has hosted life for the majority of its history (Rosing 1999; Schopf et al. 2007), hinting that liquid water has persisted for billions of years. Moreover, the geological record reveals ample evidence of extensive oceans during the Archean era, 3.8-2.5 Gya (Grotzinger \& Kasting 1993; Knoll et al. 2016). Mars also exhibits both ancient valley networks, carved out by running surface waters $\sim 4$ Gya (Wordsworth 2016), and sedimentary basins that hosted long-lived lakes (Grotzinger et al. 2015) and other large bodies of water (DiBiase et al. 2013). These features contrast sharply with Mars' modern frigid climate. Thus, from a geological perspective ancient Earth and Mars were as warm as, or warmer than, today.

Warm climates on early Earth and Mars contrast markedly with standard models of the Sun's long-term evolution. Solar luminosity is expected to have monotonically grown throughout history, such that during the Archean era, both Earth and Mars would have received between $75 \%$ and $85 \%$ as much stellar flux as today (Gough 1981; Figure 1). All else kept equal (e.g., atmospheric composition), the Earth is expected to freeze over at stellar luminosities only $\sim 10 \%$ below today (Yang et al. 2012; Hoffman et al. 2017). Given Mars' cold climate today, its ancient warmth is even more perplexing under a faint early Sun.

The apparent contradiction between the ancient Solar luminosity, and the existence of liquid water on Earth and Mars, has been dubbed the "the Faint Young Sun Paradox" (Sagan \& Mullen 1972). Most attempts at a resolution have proposed that Earth's atmosphere contained higher concentrations of greenhouse gases (Feulner 2012; Charnay et al. 2013) - varying admixtures of carbon dioxide and methane, which if present in sufficient quantities may feasibly heat the Earth to a sufficient extent (Bender 2013). These higher amounts of greenhouse gases would then be maintained within tight temperature bounds by the silicate-weathering feedback (Walker et al. 1981), with the supposed efficiency of this mechanism being extended to compute habitable zones around other stars (Kasting et al. 1993). The loss of habitability on Mars is thereby ascribed to the loss of its atmosphere, and therefore an efficient warming mechanism over time (Jakosky et al. 2018).

Models incorporating high levels of greenhouse gases have attained moderate success in reproducing a warm early Earth, but it remains challenging to precisely constrain the Archean atmospheric composition using geological data. Thus, the otherwise attractive and popular hypothesis that greenhouse gases served as a solution to the Faint Young Sun Paradox, is also at present largely unfalsifiable. The extension of the problem to Mars stretches the gap between data and theory in a fashion that places serious challenges upon greenhouse gas solutions for early Martian climate. Accordingly, it is valuable to consider other hypotheses, even if only to rule them out as alternatives.

Here, we consider the hypothesis that the evolution of luminosity derived from the standard Solar model is incorrect, because it relies on the assumption of constant mass with time. If the young Sun was a few percent more massive than at present, the stellar flux received by Earth and Mars may be maintained at similar levels to today (Bowen \& Willson 1986; Feulner 2012). This idea is decades old, but a definitive, empirical logic for falsification has not been identified (see Minton \& Malhotra 2007 for earlier attempts).

We hypothesize that if the Sun lost mass over time, it would leave a recognizable fingerprint in the characteristic orbital timescales of Earth and Mars. In this Letter, we demonstrate that the specific period of oscillation of Earth and Mars' eccentricities, driven by Milankovitch mode $g_{2}-g_{5}$, may directly constrain the Sun's mass through time to a precision of $\lesssim 1 \%$. Given that these Milankovitch parameters can be 

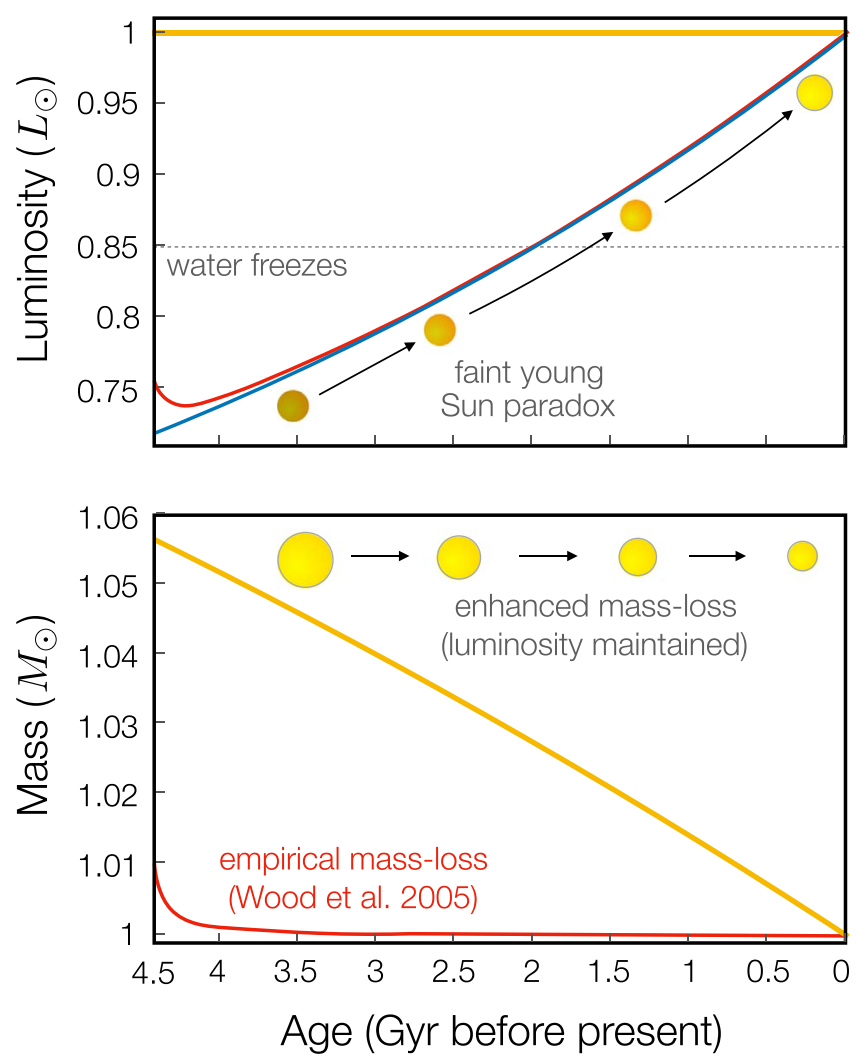

Figure 1. Time evolution of the Sun's mass and luminosity under various assumptions. In the upper panel, we present the luminosity vs. time as derived by (Gough 1981), under the assumption of a constant stellar mass (blue line) and under a mass-loss as measured for Sun-like stars (Wood et al. 2005), in addition to a constant-luminosity model (yellow). In the bottom panel, we plot the corresponding curves illustrating the time evolution of stellar mass, which is the property inferred from Milankovitch cycles in sediments.

preserved in the typical length scales recorded by sedimentary rocks, we now have a way that the mass of the ancient Sun can be observed.

\section{A Massive Young Sun}

Today, the Sun is losing mass predominantly by way of the stellar wind (in addition to a smaller, yet comparable massequivalent of photon energy). Stellar mass-loss is accompanied by an intrinsic luminosity decrease proportional to mass raised to the fourth power (Chandrasekhar 1939; Phillips et al. 1995). Concomitantly, the adiabtic invariance of orbital angular momentum causes the orbits of the planets expand in inverse proportion to the Sun's mass, denoted $M_{\star}$. When these two effects are combined, solar mass-loss causes the flux of solar irradiation received by each planet to scale as $F \propto M_{\star}^{6}$. Consequently, in order to entirely undo the expected $25 \%$ drop in luminosity, the young Sun requires $\sim 5 \%$ more mass than today.

A larger mass for the early Sun has been proposed as a contributor to ancillary mysteries, such as the Sun's lithium depletion (Graedel et al. 1991), helioseismic signatures (Guzik \& Mussack 2010), and rotational evolution (Martens 2017). These inferences of the Sun's own history are difficult to test, but attempts to measure the mass-loss from Sun-like stars might reveal crucial information regarding the Solar evolution, under the assumption that the Sun is typical.
Measurements from a small number of Sun-like stars have revealed that such stars do indeed possess stronger winds earlier in their history (Wood et al. 2005). Furthermore, there is an apparent reduction in stellar wind magnitude in stars younger than $700 \mathrm{Myr}$ that remains poorly understood. The exact magnitudes of these winds are subject to uncertainties and assumptions made in inferring their properties. Despite these issues, mass-loss rates $\dot{M}_{\star}$ derived as a function of time $t$ collectively follow (Wood et al. 2005)

$$
\dot{M}_{\star}=\dot{M}_{\odot}\left(\frac{t}{t_{0}}\right)^{-2.3},
$$

where $t_{0}=4.5 \mathrm{Gyr}$ is the approximate age of the Sun and $\dot{M}_{\odot}=2 \times 10^{-14} M_{\odot} \mathrm{yr}^{-1}$ is the current Solar wind magnitude (Phillips et al. 1995).

In Figure 1 we illustrate the inferred flux $F_{P}$ received by Earth or Mars, relative to today $F_{P, 0}$, if the Sun's mass-loss followed Equation (1) (which for the sake of illustration, we extend further back than $700 \mathrm{Myr}$ after star formation, when the observations of Wood et al. (2005) become less well understood). More specifically, we utilized the equations of Gough (1981) for the evolution of the Sun's luminosity (the term within squared parentheses below), but augmented this form by a factor of $\left(M_{\star} / M_{\odot}\right)^{6}$, such that

$$
F_{P}=F_{P, 0}\left(\frac{M_{\star}(t)}{M_{\odot}}\right)^{6}\left[1+\frac{2}{5}\left(1-\frac{t}{t_{0}}\right)\right]^{-1} .
$$

The mass-loss prescription inferred from Wood et al. (2005) is insufficient for mass-loss of more than $\sim 1 \%$, and is far short of the $\sim 5 \%$ required to entirely remove the Faint Young Sun problem (Figure 1). Furthermore, most of the mass-loss occurs early, which may be applicable to early events in the Martian record, but does little to warm Earth's lengthy Archean era, lasting until 2.5 Gya.

The massive young Sun hypothesis appears at odds with observations of Sun-like stars. Nevertheless, measurements of winds from other stars are not without substantial degrees of uncertainty (Vidotto et al. 2011). Moreover, the striking dissimilarity between our Solar System and most planetary systems hosted by other stars (Batalha et al. 2013) bolsters the case for our Sun experiencing an abnormal history of massloss. Indeed, an enhanced mass-loss may contribute to the marked absence of material interior to Mercury's orbit (Hayashi 1981; Chiang \& Laughlin 2013). A more general motivation lies in the rarity with which fundamental properties of our Solar System are observable throughout antiquity. ${ }^{3}$ If Milankovitch records reveal the ancient Sun's mass, it is important to leverage that information.

\section{Ancient Milankovitch Frequencies: Analytic Expectations}

Milankovitch cycles are defined as the quasi-periodic variations of astronomically forced insolation, first proposed as a mechanism driving Earth's past climatic variations (Milankovitch 1941). We begin with an outline of the expected dependence of Milankovitch timescales upon Solar mass, based

\footnotetext{
3 A notable example was the use of the Proterozoic-age natural nuclear reactor at Oklo, Gabon-used to constrain the ancient atomic fine structure constant (Damour \& Dyson 1996).
} 
upon analytic scaling arguments. The orbits of the eight solar system planets generally possess low inclinations and eccentricities, and their orbital periods are far from integer ratios. Consequently, the mutual gravitational interactions between planets may be approximated using a "secular" approach. Specifically, each orbit is represented as a massive wire, exerting torques upon every other wire in the system (Murray \& Dermott 1999).

The time evolution of the eccentricity and inclination of planet $i$ may be written as a linear sum of oscillatory modes $g_{i}$ (Laskar et al. 2004b, 2011a). In the linear case, the key feature of these modes is that their oscillatory frequencies scale proportionally to the planetary orbital frequencies $n_{i}$, and to the ratio of the perturbing planetary mass to the Sun's mass. Thus, the linear secular frequencies scale as

$$
g_{i} \propto n_{i}=\sqrt{\frac{G M_{\star}}{a_{i}^{3}}} \frac{1}{M_{\star}(t)},
$$

assuming constant planetary masses.

Making the assumption that the Sun lost mass slowly compared to secular timescales, the product $M_{\star} a_{i}$ remains constant for each planet (Minton \& Malhotra 2007), such that $a_{i} \propto 1 / M_{\star}$. Substituting this relationship into expression 3 yields the proportionality

$$
g_{i} \propto M_{\star}
$$

the linear mode frequencies scale proportionally with the Sun's mass.

The discussion above deals only with the frequencies, not amplitudes, of modes - amplitude estimates require numerical simulations. Furthermore, we emphasize that the solar system's orbital evolution is intrinsically chaotic, making it impossible to precisely predict orbital properties further back than 54-60 Mya (Laskar et al. 2011b; Zeebe 2017). Despite chaotic limitations, previous work has suggested a long-term stability of the mode associated with the frequency $g_{2}-g_{5}$, corresponding to a period of roughly $405 \mathrm{Kyr}$ today (Laskar et al. 2011a). Simulations $250 \mathrm{Myr}$ into the past have shown that this mode persists as a strong driver of Earth's eccentricity oscillations.

Below, we describe the results of a numerical simulation of the Solar system backward $4.5 \mathrm{Gyr}$ in time. In so doing, we tested two separate aspects of Solar System history; the first is whether or not the stability of the $g_{2}-g_{5}$ mode persists within Earth's (and Mars') orbit into more ancient times than those considered previously (Laskar et al. 2011a). Second, does its frequency scale linearly with stellar mass (Equation (4))?

\subsection{Numerical Simulations}

The linear arguments presented above ignore much complexity in the solar system's long-term evolution. The expected chaotic variations of secular modes (Laskar et al. 2011a), alongside nonlinear and general relativistic contributions reduce the confidence in a purely linear scaling between secular frequencies and the stellar mass (Bretagnon 1974). Direct numerical simulations are required to more rigorously ascertain their mutual dependence.

We performed direct $N$-body simulations of the eight solar system planets back in time $4.5 \mathrm{Gyr}$, taking between three and four weeks to complete. A WHFast symplectic algorithm was employed within the "Rebound" integrator package (details in Rein \& Liu 2012). General relativistic apsidal precession was
Table 1

The Fundamental Secular Mode Frequencies, Computed Here (Middle Column), Compared to Laskar et al. (2011a) (La2010). Modes $g_{5}-g_{8}$ Agree Well. $g_{1}-g_{4}$ Differ by $\sim 1 \%$, Likely Owing to Our Simplified Prescription for General Relativity

\begin{tabular}{lrc}
\hline \hline Mode & Value $\left({ }^{\prime \prime} \mathrm{yr}^{-1}\right)$ & La2010 $\left(^{\prime \prime} \mathrm{yr}^{-1}\right)$ \\
\hline$g_{1}$ & 5.71 & 5.60 \\
$g_{2}$ & 7.44 & 7.46 \\
$g_{3}$ & 17.19 & 17.36 \\
$g_{4}$ & 17.76 & 17.92 \\
\hline$g_{5}$ & 4.26 & 4.26 \\
$g_{6}$ & 28.25 & 28.25 \\
$g_{7}$ & 3.09 & 3.09 \\
$g_{8}$ & 0.67 & 0.67 \\
\hline
\end{tabular}

included following Nobili \& Roxburgh (1986), by way of an additional scalar potential. This prescription is simpler than that taken by Laskar et al. (2011a) and leads to a small $(\sim 0.5 \%)$ overestimation of secular frequencies $g_{1}-g_{4}$ (see Table 1) and therefore of $g_{2}-g_{5}$. Nevertheless, our primary goal is to identify the scaling between Solar mass and $g_{2}-g_{5}$, together with the mode's stability. For that, our prescription suffices.

The Sun's mass-loss rate was set at $0.05 M_{\odot}$ per $4.5 \mathrm{Gyr}$, such that the early Sun possessed 5\% more mass than today. As argued above, the true historic mass-loss rate was unlikely to be constant, but as long as the mass-loss timescale is long compared to secular oscillations, the exact form of mass loss is unimportant. As initial conditions, we extracted the current orbital position for each planet from NASA's Horizons database. ${ }^{4}$ The Earth and Moon were treated together as a single object at the pair's mutual barycenter. Finally, the simulation results were tabulated every $450 \mathrm{yr}$ in Jacobi coordinates. The full 4.5 Gyr output was split into 450 millionyear sections, within which the dominant frequencies were computed by way of a Fast Fourier Transform (Figures 2, 3) implemented in Matlab.

\section{Stability of the $g_{2}-g_{5}$ Mode}

Previous work has highlighted Earth's $g_{2}-g_{5}$ eccentricity cycle as the most stable of the Milankovitch frequencies (Laskar et al. 2011a; Hinnov 2018), meaning that it chaotically drifts least over time. These previous results demonstrated the cycle's stability during the Phanerozoic interval, but our simulations demonstrated its stability back to 4.5 Gya (Figure 3). We tested whether the $g_{2}-g_{5}$ mode frequency shifts in proportion to the Sun's mass; if so, one could envision a measurement of the Sun's ancient mass.

Figure 3 illustrates a Fourier transform of the eccentricity evolution of Earth (top panel) and Mars (bottom panel). In each plot, we present the spectrum associated with the most recent $450 \mathrm{Myr}$ section of the simulations (blue for Earth, red for Mars), alongside that for the most ancient $450 \mathrm{Myr}$ section (gray in both). The peak associated with the $g_{2}-g_{5}$ mode is highlighted; the mode shifts by $\sim 5 \%$-in proportion to the Sun's mass.

Given the aforementioned chaotic nature of the Solar system, the $5 \%$ shift of the $g_{2}-g_{5}$ mode frequency may have occurred by chance. To account for this possibility, we plotted the time

4 https://ssd.jpl.nasa.gov/horizons.cgi 


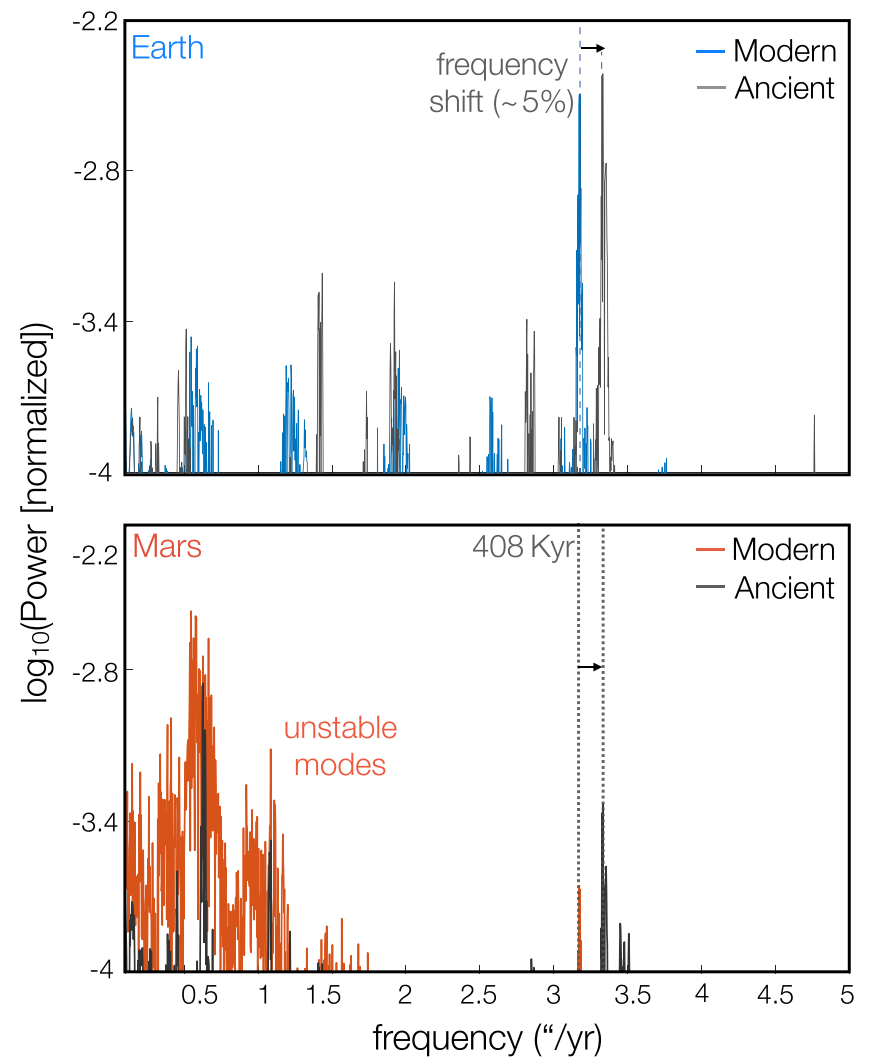

Figure 2. Fourier transform of the eccentricity evolution of Mars (bottom panel) and Earth (top panel), derived through numerical simulation. We present the most recent $450 \mathrm{Myr}$ in blue (Earth) and red (Mars), with the most ancient $450 \mathrm{Myr}$ time sequence plotted in gray (both). Notice that most modes shift somewhat, but the $g_{2}-g_{5}$ mode, labeled as " $408 \mathrm{Kyr}$," shifts most reliably by $\sim 5 \%$, i.e., by the percentage of Solar mass loss.

evolution of the $g_{2}-g_{5}$ mode within each successive $450 \mathrm{Myr}$ time period (Figure 4). Each bin is substantially longer than the $\sim 60 \mathrm{Myr}$ divergence time, over which secular modes are predictable in detail (Laskar et al. 2011b; Zeebe 2017), and so should not exhibit a predictable trend from bin to bin if the motion is dominated by chaotic drift. If, however, the time evolution is dominated by the Sun's changing mass, the period would follow a linear decrease back in time.

In Figure 3, the peak clearly shifts to higher frequencies at earlier times. The oscillation periods corresponding to the centers of these peaks are shown in Figure 4, with the linear relationship

$$
\left|g_{2}-g_{5}\right|^{-1}=408 \operatorname{Kyr}\left(\frac{M_{\star}}{M_{\odot}}\right)^{-1}
$$

superimposed. Equation (5) is the key result of this Letter, and agrees with the simulations to $\sim 1 \%$. Thus, measuring the period of the $g_{2}-g_{5}$ mode in sediments of Earth and/or Mars reveals the Sun's mass to $\sim 1 \%$.

Note that our simulations yield a modern period of $408 \mathrm{Kyr}$, whereas previous work suggested $405 \mathrm{Kyr}$. As mentioned above, this discrepancy is likely a result of our simplified treatment of relativity. In line with this explanation, we tabulated secular modes $g_{1}-g_{8}$ (Table 1). The modes associated with $g_{1}-g_{4}$ differ from Laskar et al. (2011a) by a small amount, dominate the closer-in terrestrial planets, which are more susceptible to relativistic effects. In contrast, $g_{5}-g_{8}$ agree well with these earlier results, likely reflecting the smaller effect of relativity upon the outer planets.

Mars' orbit possesses a larger eccentricity than Earth, and is physically closer to Jupiter. As such, the degree of chaos experienced by the Martian orbit is amplified relative to Earth (Laskar 1994; Laskar et al. 2004a), as can be seen by the broad range of poorly defined peaks over its most recent $450 \mathrm{Myr}$ of evolution (Figure 3). Thus, whereas both the orbits of Mars and Earth exhibit a predictable trend in the frequency associated with $g_{2}-g_{5}$, the amplitude of this mode is less predictable for Mars. In summary, the period of the $g_{2}-g_{5}$ signal on Mars and Earth is predictable, but the probability and strength of its occurrence on Mars is not.

\section{Measuring Ancient Milankovitch Frequencies}

A link between the Earth's orbital eccentricity, climate, and the banding within sediments laid down in depocenters has been suspected for centuries (Imbrie \& Imbrie 1986). These signals have now been confirmed in multiple, tens of millions of years-long paleoclimate records, spanning most of the past $250 \mathrm{Myr}$, and a large fraction of the earlier Phanerozoic (reviewed in Hinnov 2018). However, the massive young Sun hypothesis can only be directly tested with significantly older records, extending into the early Precambrian, when highquality strata become significantly rarer.

Some of the most ancient sources of suspected Milankovitch signals include shallow water carbonate platforms (Grotzinger 1986; Hofmann et al. 2004), and banded iron formations, dating back to $\sim 2.5$ Gya (Trendall et al. 2004). In particular, Milankovitch-forced signals associated with the Earth's obliquity and precession were identified within the 2.45 Gya Weeli Wolli Formation, and were used to constrain the moon-Earth separation.

More recent statistical techniques are emerging to better constrain frequencies recorded in ancient sediments, including that associated to $g_{2}-g_{5}$ (Meyers 2015; Meyers \& Malinverno 2018). A 1.4 billion year-old sequence from Xiamaling, China, was used in such a way to constrain the spin rate of the early Earth. As dating methods and statistical techniques improve, a falsification of the massive early Sun hypothesis during the Archean may soon be in reach, using Earth's sediments alone.

\subsection{Sedimentary Evidence from Mars}

Most of the Earth's ancient crust has been destroyed by plate tectonics and/or weathering. In contrast, Mars' relatively pristine, yet ancient sedimentary deposits offer a more transparent view of the Solar system's distant past. Roughly $40 \%$ of the Martian surface is older than $\sim 3.7 \mathrm{Gyr}$, with well over half exceeding $\sim 3 \mathrm{Gyr}$ in age (Solomon et al. 2005). Accordingly, most of the surface was laid down at a time during which the Sun would be expected to generate 75\%-80\% of its current luminosity at constant mass. As such, Mars may offer the most promising window in the ancient Sun's mass.

Suspected Milankovitch-forced banding of sediment has already been identified on Mars, but from orbit (Laskar et al. 2004a; Lewis et al. 2008). If future landers were equipped with precise dating techniques, most surfaces in Mars would be old enough to stand as a probe into the Sun's past. A caveat, mentioned above, is that while the timescale of the $g_{2}-g_{5}$ mode reliably tracks the Sun's mass, its magnitude is less 

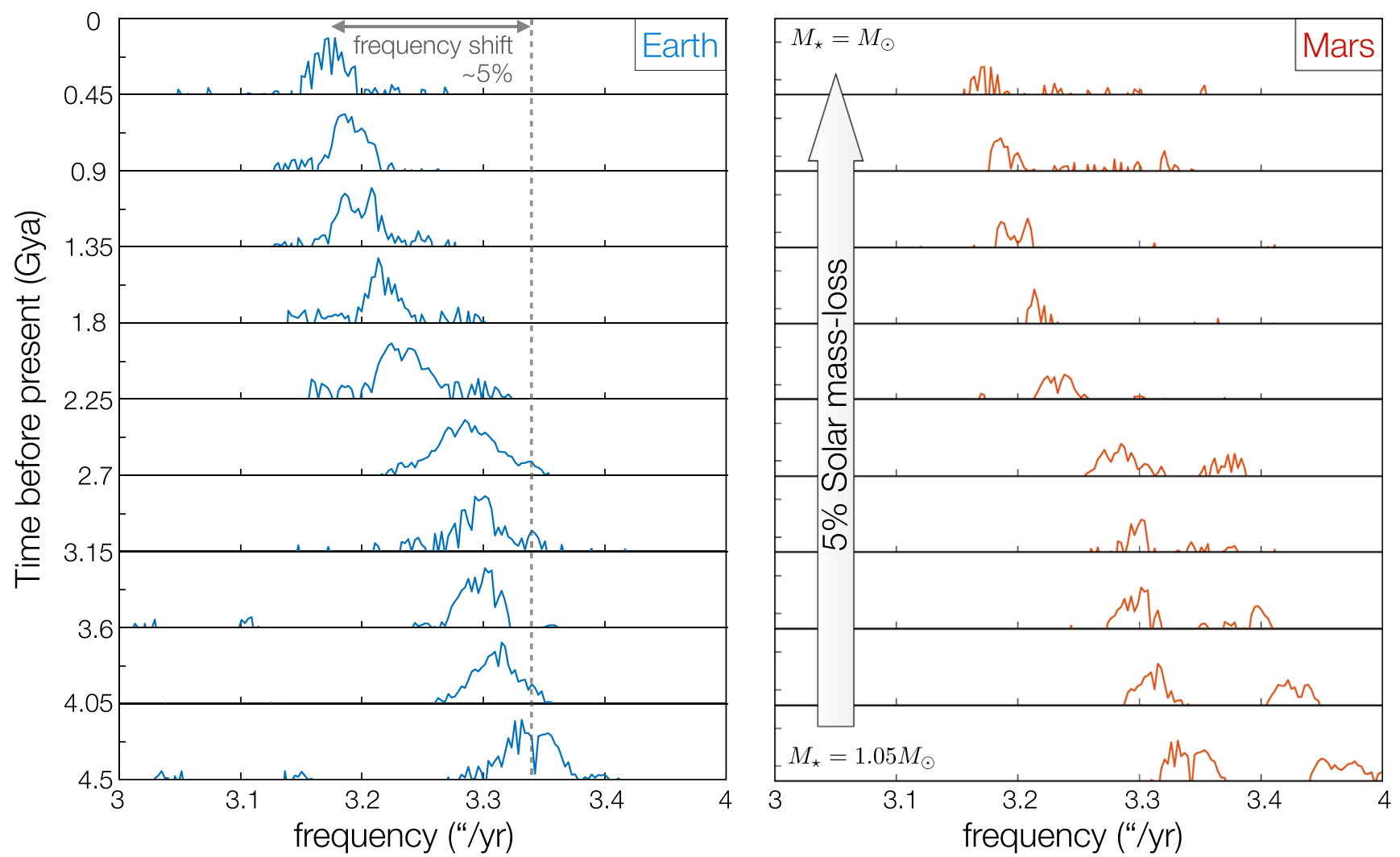

Figure 3. Fast Fourier transform spectrum of the eccentricity evolution of Earth (left panel, blue) and Mars (right panel, red), within $10450 \mathrm{Myr}$ time bins back in time. Within each panel, the $y$-axis is an arbitrary, logarithmic scale indicating the spectral power. Time runs from bottom to top, with the Sun's mass decreasing linearly from $1.05 M_{\odot}$ at the bottom to $1 M_{\odot}$ at the top.

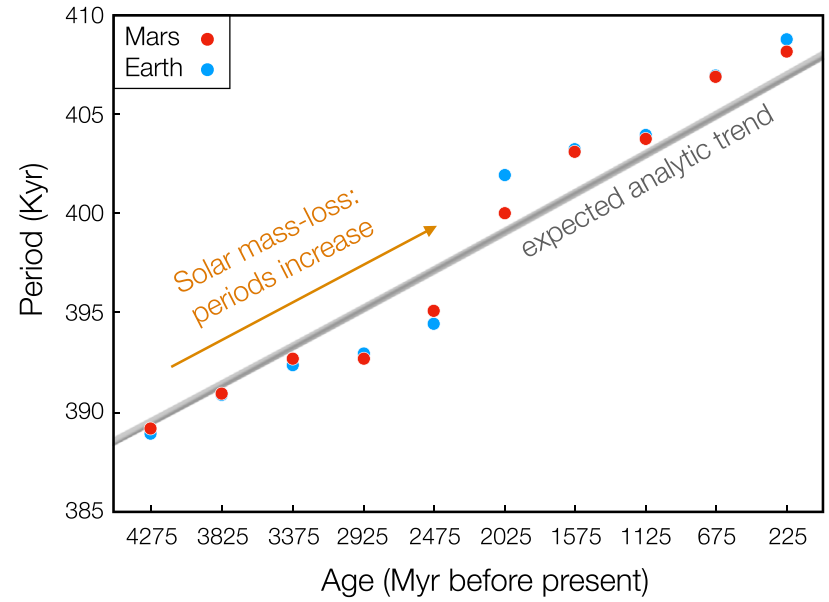

Figure 4. Time evolution of the period associated with the $g_{2}-g_{5}$ mode for Mars (red) and Earth (blue). The trend closely approximates that expected from a linear proportionality between solar mass and frequency, following Equation (5) (gray line).

predictable. Accordingly, we cannot guarantee that any given epoch will exhibit a strong $g_{2}-g_{5}$ signal, however, if one appears, its frequency scales linearly with the Sun's mass.

Aside from Milankovitch banding, a more speculative pathway toward testing the massive early Sun hypothesis is to measure the length of a year on Mars. Orbital period scales with the inverse square of Solar mass. Daily modulations in sedimentation-potentially from temperature-sensitive evaporite precipitation, or tidal rhythmites-within annual cycles would constrain the number of days per year. Given the expected constancy of the Martian day length over time, the Martian year-length would immediately follow. However, more work is needed to evaluate the feasibility of such a constraint.

\section{Conclusions}

The "Faint Young Sun Paradox"-a discrepancy between geological evidence of warm, wet early conditions on Mars and Earth, and astronomical models suggestive of a low luminosity of the early Sun-remains a central problem in Solar System history (Feulner 2012). Its resolution would have implications for the conditions persisting during the origin of life, and the potential for life to persist elsewhere in the universe.

The favored solution to the Faint Young Sun Paradox is typically that early Earth and Mars possessed thick, greenhouse gas-rich atmospheres, in order to trap more heat than today (Sagan \& Mullen 1972; Kasting et al. 1993; Charnay et al. 2013; Wordsworth 2016). However, an alternative possibility remains to be conclusively ruled out - that the Sun has lost a few percent of its mass over the previous $4.5 \mathrm{Gyr}$. In this Letter, we presented a quantitative test of this hypothesis.

Numerical simulations confirm analytic expectations that the secular frequency $g_{2}-g_{5}$ exhibits a timescale of oscillation within Earth and Mars' eccentricities that may be approximated using Equation (5); the period scales linearly with the Sun's mass. A measurement of this timescale in the sedimentary records of Earth and/or Mars would provide a direct measurement of the Sun's ancient mass. 
Put succinctly, the true history of our host star's mass over billions of years has, for the first time, entered the realm of empirical investigation.

C.S thanks Noah Planavksy and Konstantin Batygin for useful discussions. We are grateful to the referee for a thorough report that greatly improved the manuscript. This research is based in part upon work supported by NSF grant AST 1517936, NESSF Graduate Fellowship in Earth and Planetary Sciences and the 51 Pegasi b Heising-Simons Foundation grant (C.S).

\section{ORCID iDs}

Gregory Laughlin (DD https://orcid.org/0000-0002-3253-2621

\section{References}

Batalha, N. M., Rowe, J. F., Bryson, S. T., et al. 2013, ApJS, 204, 24 Bender, M. L. 2013, Paleoclimate (Princeton, NJ: Princeton Univ. Press) Bowen, G. H., \& Willson, L. A. 1986, Metic, 21, 338

Bretagnon, P. 1974, A\&A, 30, 141

Chandrasekhar, S. 1939, C\&T, 55, 412

Charnay, B., Forget, F., Wordsworth, R., et al. 2013, JGRD, 118, 10414

Chiang, E., \& Laughlin, G. 2013, MNRAS, 431, 3444

Damour, T., \& Dyson, F. 1996, NuPhB, 480, 37

DiBiase, R. A., Limaye, A. B., Scheingross, J. S., Fischer, W. W., \& Lamb, M. P. 2013, JGRE, 118, 1285

Feulner, G. 2012, RvGeo, 50, RG2006

Gough, D. 1981, Physics of Solar Variations (Berlin: Springer), 21

Graedel, T., Sackmann, I.-J., \& Boothroyd, A. 1991, GeoRL, 18, 1881

Grotzinger, J. 1986, PalOc, 1, 403

Grotzinger, J., Gupta, S., Malin, M., et al. 2015, Sci, 350, aac7575

Grotzinger, J. P., \& Kasting, J. F. 1993, JG, 101, 235

Guzik, J. A., \& Mussack, K. 2010, ApJ, 713, 1108

Hayashi, C. 1981, PThPS, 70, 35

Hinnov, L. A. 2018, Cyclostratigraphy and Astrochronology, 3, 1

Hoffman, P. F., Abbot, D. S., Ashkenazy, Y., et al. 2017, SciA, 3, e1600983

Hofmann, A., Dirks, P. H., \& Jelsma, H. A. 2004, JSedR, 74, 64
Imbrie, J., \& Imbrie, K. P. 1986, Ice Ages: Solving the Mystery (Cambridge, MA: Harvard Univ. Press)

Jakosky, B., Brain, D., Chaffin, M., et al. 2018, Icar, 315, 146

Kasting, J. F., Whitmire, D. P., \& Reynolds, R. T. 1993, Icar, 101, 108

Knoll, A. H., Bergmann, K. D., \& Strauss, J. V. 2016, RSPTB, 371, 20150493

Laskar, J. 1994, A\&A, 287, L9

Laskar, J., Correia, A., Gastineau, M., et al. 2004a, Icar, 170, 343

Laskar, J., Fienga, A., Gastineau, M., \& Manche, H. 2011a, A\&A, 532, A89

Laskar, J., Gastineau, M., Delisle, J.-B., Farrés, A., \& Fienga, A. 2011b, A\&A, 532, L4

Laskar, J., Robutel, P., Joutel, F., et al. 2004b, A\&A, 428, 261

Lewis, K. W., Aharonson, O., Grotzinger, J. P., et al. 2008, Sci, 322, 1532

Martens, P. C. 2017, in IAU Symp. S328, Living Around Active Stars, ed. D. Nandy et al. (Cambridge: Cambridge Univ. Press), 350

Meyers, S. R. 2015, PalOc, 30, 1625

Meyers, S. R., \& Malinverno, A. 2018, PNAS, 115, 6363

Milankovitch, M. 1941, Kanon der Erdbestrahlung und seine Anwendung auf das Eiszeitenproblem (Belgrade: Serbian Academy of Arts and Sciences)

Minton, D. A., \& Malhotra, R. 2007, ApJ, 660, 1700

Murray, C. D., \& Dermott, S. F. 1999, Solar System Dynamics (Cambridge: Cambridge Univ. Press)

Nobili, A., \& Roxburgh, I. W. 1986, in IAU Symp. 114, Relativity in Celestial Mechanics and Astrometry: High Precision Dynamical Theories and Observational Verifications, ed. J. Kovalevsky \& V. A. Brumberg (Dordrecht: Springer), 105

Phillips, J., Bame, S., Barnes, A., et al. 1995, GeoRL, 22, 3301

Rein, H., \& Liu, S.-F. 2012, A\&A, 537, A128

Rosing, M. T. 1999, Sci, 283, 674

Sagan, C., \& Mullen, G. 1972, Sci, 177, 52

Schopf, J. W., Kudryavtsev, A. B., Czaja, A. D., \& Tripathi, A. B. 2007, PreR, 158,141

Solomon, S. C., Aharonson, O., Aurnou, J. M., et al. 2005, Sci, 307, 1214

Trendall, A., Compston, W., Nelson, D., De Laeter, J., \& Bennett, V. 2004, AuJES, 51, 621

Vidotto, A., Jardine, M., Opher, M., Donati, J., \& Gombosi, T. 2011, MNRAS, 412,351

Walker, J. C., Hays, P., \& Kasting, J. F. 1981, JGRC, 86, 9776

Wood, B. E., Müller, H.-R., Zank, G. P., Linsky, J. L., \& Redfield, S. 2005 , ApJL, 628, L143

Wordsworth, R. D. 2016, AREPS, 44, 381

Yang, J., Peltier, W., \& Hu, Y. 2012, CliPa, 8, 907

Zeebe, R. E. 2017, AJ, 154, 193 